\title{
Recaídas en incapacidad temporal: impacto de su regulación y control
}

\section{Relapses Into Temporary Disability: Impact of its Regulation and Control}

\author{
Luis Sánchez Galán', Pilar Baides Gonzalvo', Raúl Regal Ramos' \\ 1. Instituto Nacional de la Seguridad Social. Unidad Médica de la Dirección Provincial. Madrid. España. \\ Recibido: 14-09-2019 \\ Aceptado: 19-09-2019
}

\section{Correspondencia}

Luis Sánchez galán

Correo electrónico: Luis.sanchez@seg-social.es

Resumen

Tras el alta médica finaliza un proceso de incapacidad temporal. se considera que existe recaída en un mismo proceso de incapacidad temporal cuando se produce una nueva baja médica, por la misma o similar patología, dentro de los ciento ochenta días naturales siguientes a la fecha de efectos del alta médica anterior. Cuando estas recaídas ocurren tras altas médicas emitidas por el Instituto Nacional de la Seguridad Social española quedan sometidas a especiales requerimientos reglamentarios que consisten en un sistema de inspección directa llevada a cabo por los inspectores médicos adscritos a la Seguridad Social, así como por los Equipos de Valoración de Incapacidades. Las recaídas afectan directamente a la delimitación de la duración máxima del subsidio de incapacidad temporal en cada trabajador. Su número, y por tanto el gasto económico que ocasionan al sistema de seguridad social, puede disminuir al colaborar con los Servicios de Prevención de las empresas y su control puede verse favorecido aplicando modelos de inteligencia artificial.

Med Segur Trab (Internet). 2019;65(256):217-232

Palabras clave: incapacidad laboral, recaída, inteligencia artificial, Seguridad Social

\begin{abstract}
A process of temporary disability concludes once a medical discharge takes place. A relapse in the same temporary disability process is considered to take place when a new sick leave happens because of the same or a similar pathology within the 180 calendar days following the previous sick leave. If these relapses appear after the medical discharge issued by the Spanish Social Security National Institute, they will be submitted to special statutory requirements consisting of a direct inspection system carried out by Social Security medical inspectors as well as by the Disabilities Assessment Board. Relapses affect directly the definition of the maximum duration of temporary incapacity benefit for each worker. The number of such workers, and thus the Social Security economic costs are lowered by collaborating with
\end{abstract}

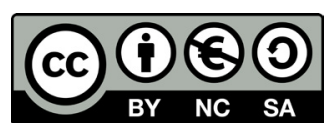

https://creativecommons.org/licenses/by-nc-sa/4.0/ 
the Prevention Services of companies. Its control could benefit from applying artificial intelligence models.

Med Segur Trab (Internet). 2019;65(256):217-232

Keywords: sick leave, recurrence, relapse, artificial intelligence, social security 


\section{LA INCAPACIDAD TEMPORAL (IT) EN LA HISTORIA DE LA MEDICINA}

Es interesante leer a Laín Entralgo describiendo la Historia de la Medicina, y ver que, junto a otros autores, afirma que de la misma forma que la anatomía y la fisiología fueron los fundamentos de la medicina clásica, y de igual forma que la física y la química fueron las ciencias básicas de la medicina del siglo XIX, las disciplinas ecológicas son los pilares de la medicina del tercer milenio: una Antropología médica basada sobre la Psicología y la Pedagogía social, una Sociología y un Derecho médicos, una Higiene general, y tal vez una Historia de la medicina, capaz de colocar las piedras sillares para una obligatoria Teoría de la Medicina ${ }^{1}$.

En este contexto médico del tercer milenio la medicina legal contemporánea ha experimentado un triple proceso: se ha incrementado el contenido técnico de ella a favor del avance general de las técnicas modernas; se han formado cuerpos de doctrina relativamente autónomos (toxicología, medicina del trabajo); se ha acentuado la orientación social de la teoría y la práctica de la disciplina (lesiones y accidentes laborales, proyección legal de cuestiones éticas, peritajes psiquiátricos, etc.).

Entre esos procesos se ha desarrollado la medicina evaluadora entendida como aquella actividad médica específica que estudia las diferentes enfermedades o trastornos que padece una persona, a efectos de determinar su magnitud y su repercusión, sobre diferentes aspectos de la vida de esa persona o de la relación de ésta con el entorno social $^{2}$. La medicina evaluadora es pues una medicina aplicada, orientada fundamentalmente a resolver problemas prácticos como es el caso de determinar las limitaciones orgánicas y funcionales para el desempeño de los requerimientos físicos y psíquicos de una actividad laboral, fuera del marco de la medicina asistencial.

Esta determinación del impedimento o menoscabo para el trabajo aparece ya reflejada para muchas enfermedades relacionadas con el trabajo en la obra de Bernardo Ramazzini. Así en su clásico "Tratado de las Enfermedades de los Artesanos" ya viene aconsejando el abandono de algunas clases de arte en función de la expresión de las enfermedades con sus síntomas o signos. Sirva de ejemplo cómo en el capítulo XXXVIII cuando habla "de las enfermedades que suelen afectar a los recitadores, a los cantores y a otros del mismo género" señala que "cuando los amanece algún mal del pecho, lo que podrá diagnosticarse por la tos y el aspecto del cuerpo, se deberá aconsejar el abandono de esta clase de arte"3.

Este abandono de la actividad laboral que ya se prescribía por el médico en el año 1700 se ha convertido en los estados de bienestar del tercer milenio en la figura remunerada de la incapacidad laboral, temporal o permanente, derivado de la contingencia que corresponda, en la que confluyen aspectos sanitarios, y por otro lado aspectos laborales, económicos y sociales, que provocan una serie de importantes consecuencias que afectan principalmente ${ }^{4}$ :

- Al trabajador, que sufre la alteración de la salud que le imposibilita el desempeño efectivo de su trabajo, y ve reducida su capacidad de ingresos.

- A la empresa, que durante las situaciones de ausencia del trabajador ve reducidos sus recursos y su productividad.

- Al Sistema de Seguridad Social, que asume el coste al sufragar al trabajador con una renta de carácter contributivo y sustitutiva del salario no percibido derivado de la situación de baja médica.

Efectivamente la dimensión económica de la determinación del menoscabo laboral ya sea temporal o permanente, en un estado del bienestar del siglo XXI, como es el español, explica la necesidad de un ordenamiento jurídico y una prestación económica sometida al mayor rigor posible, tanto en su contexto de prescripción sanitaria, como en el de inspección médica de la duración y procedencia de dicha prescripción. 


\section{LA INCAPACIDAD TEMPORAL EN EL GASTO DE LA SEGURIDAD SOCIAL ESPAÑOLA}

Los presupuestos generales del estado prorrogados para 2019 arrojan un presupuesto de gasto para prestaciones económicas de la seguridad social de 142.040.818.200€, siendo del ámbito contributivo 130.723.467.810. Incluidos en la cifra anterior, corresponde un presupuesto para la prestación de IT de 7.979.720.000 € y 12.381.747.610 € para el ámbito contributivo de la prestación de incapacidad permanente (IP). La incapacidad laboral significa por tanto un $17 \%$ del presupuesto en pensiones del sistema de seguridad social contributivo5.

Este presupuesto en pensiones se puede considerar muy importante en las cuentas estatales de España, donde el conjunto del año 2018, el producto interior bruto (PIB) a precios corrientes se sitúa en 1.208.248 millones de euros, un 3,6\% superior al de 2017. En términos de volumen, el PIB registra un crecimiento del $2,6 \%{ }^{6}$.

En este contexto de cifras macroeconómicas conviene centrarse en la incapacidad temporal y hacer una aproximación a una escala más próxima. Por ejemplo, a nivel de un Centro de Salud. Se admite que en la representación de los gastos anuales que genera un centro de Atención Primaria de tamaño medio (18.000 habitantes), la partida económica de la incapacidad temporal representa el $50,6 \%$, seguido de la farmacia $(31,8 \%)$ y de los gastos de personal $(15,9 \%)^{7}$. El sentido común por tanto obliga a reconocer que la incapacidad temporal y la farmacia, en este orden, deben ser las principales áreas de gestión, a pesar de las diferentes fuentes de esta financiación: la primera es financiada por la seguridad social y la segunda por el presupuesto sanitario de cada Comunidad Autónoma (CCAA).

Desde el año 2013 hasta el año 2019 se viene produciendo una elevación importante de los índices que reflejan la situación de uso de la prestación de IT (datos de contingencias comunes excepto autónomos):

- La duración media de los procesos de incapacidad temporal en el año 2013 era de 36,06 días y en el año 2018 fue de 38,72 días.

- La incidencia media mensual por cada mil trabajadores protegidos fue de 18,19 en 2013 y de 24,96 en 2018.

- El número medio mensual de procesos iniciados en 2013 era de 262.345 y en el año 2018 fue de 403.485

Este cuadro general de aumento del consumo de la prestación de IT $^{8,9}$ requiere que los mecanismos de control para el buen uso de la prestación establecidos en la normativa vigente de seguridad social sean óptimos, así como la necesaria delimitación de su duración máxima.

La repercusión económica de esta prestación es de gran magnitud en la sociedad no sólo por los importes de lo presupuestado para hacer frente a los gastos de esta prestación, sino por la pérdida de oportunidad que supone el gasto en IT. El informe Adecco referido a 2017, cifra el coste de oportunidad, tomando como referencia el PIB para 2017, de forma que el valor de los bienes y servicios que se habrían dejado de producir y prestar, para ese equivalente de trabajadores protegidos por el subsidio de IT, ascendió a 63.577,16 millones de euros (un 5,46\% del PIB), para el ejercicio $2017^{10}$.

\section{LA INCAPACIDAD TEMPORAL EN EL CONTROL MÉDICO DE PRESTACIONES ECONÓMICAS}

La incapacidad temporal afecta al año sólo al 30\% de la población activa. La población activa representa el $30 \%$ de la población total. Por tanto, la situación de incapacidad 
temporal afecta a algo más del $10 \%$ de la población. Si un cupo médico fuera de 2000 personas, la IT se centra en 200 personas cada $\mathbf{a n ̃ o}^{7}$.

En esta línea los médicos reconocen que de los días consumidos en situación de incapacidad temporal por el $10 \%$ de la población real del país, un tercio parece ser superfluo. De estos días superfluos, un tercio se debe a problemas del sistema, otro tercio se debe a simuladores y otro tercio a problemas complejos ${ }^{7}$.

Los estudios de la Sociedad Española de Medicina Familiar y Comunitaria (SEMFYC) demuestran que la IT, agrupada por duración, se comporta igual que la llamada Ley de Pareto o ley ABC. Esta ley viene a decir que el 20\% de los sucesos generan el $80 \%$ de los consumos medidos en días o en euros, por lo que si identificamos ese $20 \%$ de las IT (las de mayor duración) y las controlamos influiremos sobre el $80 \%$ del gasto, con sólo el 20\% del esfuerzo ${ }^{7}$.

Pero el control de la incapacidad temporal de corta duración también es relevante. El mencionado informe Adecco ${ }^{10}$ viene a recordar la posibilidad de control que tiene la empresa en esta materia aplicando el artículo 20.4 del Estatuto de los Trabajadores: El empresario podrá verificar el estado de enfermedad o accidente del trabajador que sea alegado por éste para justificar sus faltas de asistencia al trabajo, mediante reconocimiento a cargo de personal médico. La negativa del trabajador a dichos reconocimientos podrá determinar la suspensión de los derechos económicos que pudieran existir a cargo del empresario por dichas situaciones ${ }^{11}$. Este mecanismo de control en las bajas médicas de corta duración y ante posibles recaídas o recidivas de una situación de incapacidad temporal puede ser de interés, sobre todo cuando se produzca en colaboración con la inspección médica de la administración de la Seguridad Social, competente para emitir altas médicas por inspección y recientemente también para acumular procesos de IT en su caso.

Para un eficaz control médico de la IT dentro de las prestaciones económicas de la seguridad social, los inspectores médicos del INSS tienen la competencia para emitir altas médicas desde el año 2011, después de que la disposición adicional decimonovena de la Ley 35/2010, de 17 de septiembre, de medidas urgentes para la reforma del mercado de trabajo, modificara la Ley General de Seguridad Social vigente en ese momento, texto refundido aprobado por el Real Decreto Legislativo 1/1994, de 20 de junio, añadiendo una nueva disposición adicional con el siguiente contenido: "Disposición adicional quincuagésima segunda. Competencias sobre los procesos de Incapacidad Temporal ${ }^{12}$.

Hasta el cumplimiento de la duración máxima de trescientos sesenta y cinco días de los procesos de incapacidad temporal del Sistema de la Seguridad Social, el Instituto Nacional de la Seguridad Social, y, en su caso, el Instituto Social de la Marina, a través de los Inspectores Médicos adscritos a dichas entidades, ejercerán las mismas competencias que la Inspección de Servicios Sanitarios de la Seguridad Social u órgano equivalente del respectivo Servicio Público de Salud, para emitir un alta médica a todos los efectos. Cuando el alta haya sido expedida por el Instituto Nacional de la Seguridad Social o el Instituto Social de la Marina, éstos serán los únicos competentes, a través de sus propios médicos, para emitir una nueva baja médica en la situación de incapacidad temporal si aquélla se produce en un plazo de ciento ochenta días siguientes a la citada alta médica por la misma o similar patología.

La Secretaría de Estado de la Seguridad Social, a propuesta del Instituto Nacional de la Seguridad Social, y mediante resolución publicada en el Boletín Oficial del Estado, determinará la fecha a partir de la cual se asumirán las funciones atribuidas en el párrafo anterior.» Esta regulación fue desarrollada por Resolución de 15 de noviembre de 2010, de la Secretaria de Estado de la Seguridad Social.

En la actualidad es el artículo 170.1 de la Ley General de la Seguridad Social ${ }^{13}$ donde se recoge esta facultad de emitir el alta médica desde el INSS a través de sus inspectores médicos. 
Además es necesario destacar que esta Ley ${ }^{13}$ provee de mecanismos especiales de control para poder iniciar procesos consecutivos de incapacidad temporal en aquellos casos en los que ha existido la emisión previa de un alta médica por el Instituto Nacional de la Seguridad Social (INSS), abriendo la posibilidad de ser considerados como un único proceso de incapacidad temporal, de cara a mantener en todo caso la duración máxima establecida para este subsidio, respetando los principios de proporcionalidad y eficacia que rigen en materia de seguridad social.

Las altas médicas del INSS que requieren dictamen del Equipo de Valoración de Incapacidades, por haber superado los 365 días de IT y las resoluciones de denegación de incapacidad permanente tras superar los 545 días constituyen también otros dos puntos de partida para ahondar el control de las nuevas bajas consecutivas a estas resoluciones que suponen la reincorporación del trabajador a su actividad laboral.

\section{CONCEPTOS LEGALES DE INCAPACIDAD TEMPORAL Y RECAÍDA DE INCAPACIDAD TEMPORAL}

El Texto Refundido de la Ley General de la Seguridad Social ${ }^{13}$ dedica el artículo 169 a definir el concepto de incapacidad temporal, de forma que tendrán la consideración de situaciones determinantes de incapacidad temporal:

a) Las debidas a enfermedad común o profesional y a accidente, sea o no de trabajo, mientras el trabajador reciba asistencia sanitaria de la Seguridad Social y esté impedido para el trabajo, con una duración máxima de trescientos sesenta y cinco días, prorrogables por otros ciento ochenta días cuando se presuma que durante ellos puede el trabajador ser dado de alta médica por curación.

b) Los períodos de observación por enfermedad profesional en los que se prescriba la baja en el trabajo durante los mismos, con una duración máxima de seis meses, prorrogables por otros seis cuando se estime necesario para el estudio y diagnóstico de la enfermedad.

A efectos del período máximo de duración de la situación de incapacidad temporal que se señala en la letra a) del apartado anterior, y de su posible prórroga, se computarán los períodos de recaída y de observación.

Se expone en el mencionado artículo que se considerará que existe recaída en un mismo proceso cuando se produzca una nueva baja médica por la misma o similar patología dentro de los ciento ochenta días naturales siguientes a la fecha de efectos del alta médica anterior.

De la definición legal anterior se concluyen dos condiciones necesarias para que un proceso de incapacidad temporal pueda ser considerado como recaída:

En primer lugar, el proceso de incapacidad temporal debe iniciarse en un plazo no superior a 180 días con respecto al alta emitida en el proceso último anterior del cuál es recaída.

En segundo lugar, el proceso de incapacidad temporal debe originarse por la misma o similar patología del proceso último anterior del cuál es recaída.

El Instituto Nacional de la Seguridad Social (INSS) interpreta que es de aplicación el criterio etiológico para aclarar el concepto de "misma o similar patología", de forma que en el plazo temporal establecido, cuando se demuestra una misma etiología de la enfermedad, se debe asumir que se trata de la misma o de similar enfermedad.

Determinados autores ${ }^{14}$ exponen las dos posturas enfrentadas existentes con carácter previo al concepto actual de recaída. De una parte, estaba la opinión de primar el aspecto temporal considerando que procede la acumulación de procesos de IT en el supuesto de que un trabajador vuelva a enfermar por las mismas o distintas o dolencias y no hayan 
transcurrido más de 6 meses y de la otra, los que postulaban que tan sólo es posible aunar ambos procesos cuando la enfermedad sea debida a la misma enfermedad.

Finalmente, el Tribunal Supremo (TS) en Unificación de Doctrina de 8 de mayo de 1995, establece que, si no han transcurrido más de seis meses de actividad laboral y se trata de la misma dolencia, entonces sí procede la acumulación de procesos.

A pesar de todo algunos autores en el año 2007 consideraron necesario advertir a este respecto que uno de los principios de nuestro sistema, cual es la "conjunta consideración de las contingencias protegidas", tan complejo en su ordenación, como olvidado o inaplicado en la práctica es clave de equilibro entre la garantía del derecho y la racionalidad de su aplicación ${ }^{15}$.

En materia competencial, diciembre de 2018 ha sido la última fecha en que se da una nueva redacción al artículo 170.1 de la Ley General de la Seguridad Social para exponer que el INSS ejercerá, a través de los inspectores médicos adscritos a dicha entidad, las mismas competencias que la Inspección de Servicios Sanitarios de la Seguridad Social u órgano equivalente del respectivo servicio público de salud, para emitir un alta médica a todos los efectos, así como para considerar que existe recaída en un mismo proceso cuando se produzca una nueva baja médica por la misma o similar patología dentro de los ciento ochenta días naturales siguientes a la fecha de efectos del alta médica anterior $^{16}$.

Este reconocimiento de las recaídas facilitará la gestión de la incapacidad temporal al permitir al inspector médico del INSS acumular diversos procesos de incapacidad temporal que habían sido objeto de baja y/o alta, en su caso, no solo por el inspector médico, sino también cuando había sido emitida el alta médica por el médico de atención primaria.

\section{ASPECTOS JURÍDICOS DESTACADOS EN LAS RECAÍDAS DE INCAPACIDAD TEMPORAL DE PROCESOS FINALIZADOS POR EL INSS. RECAÍDAS DESPUÉS DE RESOLUCIONES DE ALTA MÉDICA Y RECAÍDAS DESPUÉS DE RESOLUCIONES DE "NO CAUSA DE INCAPACIDAD PERMANENTE"}

Un primer aspecto jurídico previo a la definición anteriormente expuesta de las recaídas, consiste en que las normas han venido estableciendo un límite temporal para la consideración de un proceso de incapacidad temporal recaída de otro previo, de tal forma que la Orden de 13 de octubre de 1967 por la que se establecen normas para la aplicación y desarrollo de la prestación por incapacidad laboral transitoria en el Régimen General de la Seguridad Social establece en su artículo 9 la duración del derecho y concretamente afirma que si el proceso de incapacidad laboral transitoria se viere interrumpido por períodos de actividad laboral por un tiempo superior a seis meses, se iniciará otro nuevo, aunque se trate de la misma o similar enfermedad ${ }^{17}$.

Queda pues el concepto de recidiva para aquellos procesos de incapacidad temporal que obedecen a una misma enfermedad, pero entre los cuales queda una distancia superior a 6 meses.

Otro antecedente jurídico en materia de recaídas de significativa relevancia que establece la condición particular de recaída tras la emisión del alta médica por el inspector médico de la administración de la Seguridad Social viene dado por la Orden de 21 de marzo de 1974, por la que se regulan determinadas funciones de la Inspección de Servicios Sanitarios de la Seguridad Social en materia de altas médicas ${ }^{18}$.

Esta Orden establece en su artículo primero que la Inspección de Servicios Sanitarios de la Seguridad Social, de oficio o en virtud de la información recibida de las Empresas o de los Servicios Médicos de las mismas, podrá decretar, previas las actuaciones que estime procedentes, el alta médica de los trabajadores que se encontraran en situación de 
incapacidad laboral transitoria debida a enfermedad común o accidente no laboral. El alta médica que se decrete de acuerdo con lo previsto anteriormente determinará la extinción de la situación de incapacidad laboral transitoria, conforme a lo establecido en el apartado a) del número 1 del artículo 10 de la Orden de 13 de octubre de 1967. Lo dispuesto previamente se entenderá sin perjuicio de que los Servicios Sanitarios de la Seguridad Social continúen prestando al trabajador la asistencia sanitaria que, sin requerir una nueva baja médica, aconseje su estado.

El artículo segundo de la mencionada Orden de 1974 indica que, durante un periodo, que será determinado por la Inspección de Servicios Sanitarios y que no podrá ser inferior a seis meses, la baja médica de los trabajadores que hayan sido dados de alta de conformidad con lo dispuesto en el artículo anterior corresponderá a dicha Inspección.

En el momento actual es el Real Decreto 625/2014, de 18 de julio ${ }^{19}$, el que regula determinados aspectos de la gestión y control de los procesos por IT en los primeros trescientos sesenta y cinco días de su duración. Establece en su artículo 7.6 que cuando en un proceso de IT se haya expedido el parte médico de alta por el INSS o, en su caso, por el Instituto Social de la Marina, a través de los inspectores médicos adscritos a dichas entidades, durante los ciento ochenta días naturales siguientes a la fecha en que se expidió el alta, serán estas entidades las únicas competentes, a través de sus propios médicos, para emitir una nueva baja médica por la misma o similar patología.

En suma, cuando el INSS ha finalizado un proceso de IT, el texto refundido de la Ley General de Seguridad Social de 2015 expone tres situaciones de recaída posibles para ese proceso de IT, antes de que transcurran 180 días desde su finalización:

1. Recaídas contempladas en el artículo 170.1, es decir aquellas bajas médicas que se producen tras la emisión de altas médicas por el inspector médico del INSS, en procesos de incapacidad temporal que no superen los 365 días de duración.

2. Recaídas contempladas en el artículo 170.2, es decir aquellas bajas médicas que se producen tras la emisión de altas médicas por el director provincial del INSS.

3. Recaídas contempladas en el artículo 174, es decir aquellas bajas médicas que se producen tras la denegación de incapacidad permanente, se hayan agotado o no previamente los 545 de incapacidad temporal.

El último aspecto jurídico destacado ahora en las recaídas acontece por la diferenciación del concepto de recaída (mismo proceso de incapacidad temporal por no haberse agotado el periodo máximo de duración en su caso, no haberse producido actividad laboral intermedia en un periodo superior a 6 meses y ser similar patología causante de la baja)) y recidiva (distinto proceso de incapacidad temporal ya sea por diferente causa, por agotamiento del subsidio, o por haber transcurrido más de 6 meses), y se refiere a la diferenciación de los requisitos para acceder al subsidio, así como una diferencia en la entidad pagadora.

Para el supuesto que hemos denominado recaída en un mismo proceso de incapacidad temporal el hecho causante de las mismas ha de situarse en la fecha en la cual se produjo la baja inicial, de manera que es en esta fecha de la baja inicial a la que habrá que referir el cumplimiento o concurrencia de los requisitos exigibles de alta y de carencia contemplados en los artículos 172-174 de la Ley General de Seguridad social (situación de alta o asimilada y en caso de enfermedad común, ciento ochenta días dentro de los cinco años inmediatamente anteriores al hecho causante).

En el supuesto que hemos denominado recidiva resulta exigible la concurrencia general de todos los requisitos expuestos en la fecha de la correspondiente baja médica por la misma o similar patología, por ser de aplicación los artículos 172 y 174 de la Ley General de la Seguridad Social.

Esta diferencia entre ambas situaciones de requerimientos se fundamenta en la idea de la situación de "recaída" como un "periodo único de incapacidad temporal", en el cual 
los requisitos exigidos en la fecha de la baja inicial conservan toda su virtualidad en la segunda baja médica o sucesiva. Sin embargo, en los supuestos de "recidivas" (por haberse producido entre ambas situaciones un periodo superior a seis meses) nos encontramos ante un proceso distinto donde los requisitos exigidos para acceder a la prestación de incapacidad temporal han de cumplirse en la fecha de la nueva baja.

\section{ASPECTOS MÉDICOS DESTACADOS DE LAS RECAÍDAS DE INCAPACIDAD TEMPORAL, CON ESPECIAL ATENCIÓN A LAS RECAÍDAS TRAS LAS ALTAS MÉDICAS EMITIDAS POR LOS INSPECTORES MÉDICOS DEL INSS (170.1). DATOS DE RECAÍDA TRAS ALTA MÉDICA EMITIDA POR LOS DIRECTORES PROVINCIALES DEL INSS EN PRIMERA RESOLUCIÓN (170.2). DATOS DE RECAÍDAS TRAS LA DENEGACIÓN DE INCAPACIDAD PERMANENTE (ARTÍCULO 174)}

En primer lugar y en cuanto a las bajas médicas por recaída contempladas en el artículo 170.1, el inspector médico del INSS valora cada caso concreto, a través del Aplicativo de Trabajo Informático de las Unidades Médicas (ATRIUM), ya sean trabajadores que han solicitado ellos mismos la baja por recaída en un Centro de Atención e Información de la Seguridad Social (CAISS), o bien sean trabajadores con cualquier baja médica emitida por el médico de atención primaria, en ambos casos, en el periodo de 180 días naturales posteriores a la emisión de un alta médica por el inspector médico del INSS.

Cabe señalar, por la experiencia de los autores, que las altas médicas emitidas por los inspectores médicos del INSS son más frecuentes en torno a los 180 días de duración de los procesos de IT.

Tras esta valoración el inspector médico puede adoptar cuatro posibles decisiones:

1. Denegación de la baja médica por similar patología previo reconocimiento médico expreso.

2. Emisión de baja médica por similar patología.

3. Resolución como distinta patología a la del proceso finalizado anteriormente, donde puede proceder la baja médica a criterio último de su médico de atención primaria.

4. Resolución de distinta patología sin que impresione la necesidad de iniciar una baja médica.

De esta forma ya en el año 2011 se emitieron 36.126 altas médicas, correspondientes a un 10,20\% de los 354.088 reconocimientos médicos realizados en las Unidades Médicas del INSS de procesos de IT, lo que evidenció la oportunidad de la medida entonces adoptada ${ }^{20}$.

Si de forma más actualizada, de los años comprendidos entre ese año 2011 y el año 2019 actual, tomamos los datos del año 2017, observamos una mejora gradual de la eficiencia de los reconocimientos médicos realizados observando que ese año 2017 se efectuaron 155.820 reconocimientos médicos por los médicos evaluadores del INSS, emitiéndose 28.900 altas médicas por los mencionados inspectores (porcentaje: $18,55 \%)^{21}$

Frente a estas 28.900 altas médicas por inspección han existido 8.982 casos de posibles recaídas, ya sea por solicitud de baja del interesado, o bien por valoración desde el INSS de una baja emitida por el médico de familia ${ }^{22}$.

De los cuatro posibles resultados a lo largo de los años se obtiene el siguiente gráfico: 


\section{Gráfico 1. Procesos de recaída tras alta emitida por el inspector médico del INSS} RECAÍDAS ALTAS INSP MÉDICA INSS

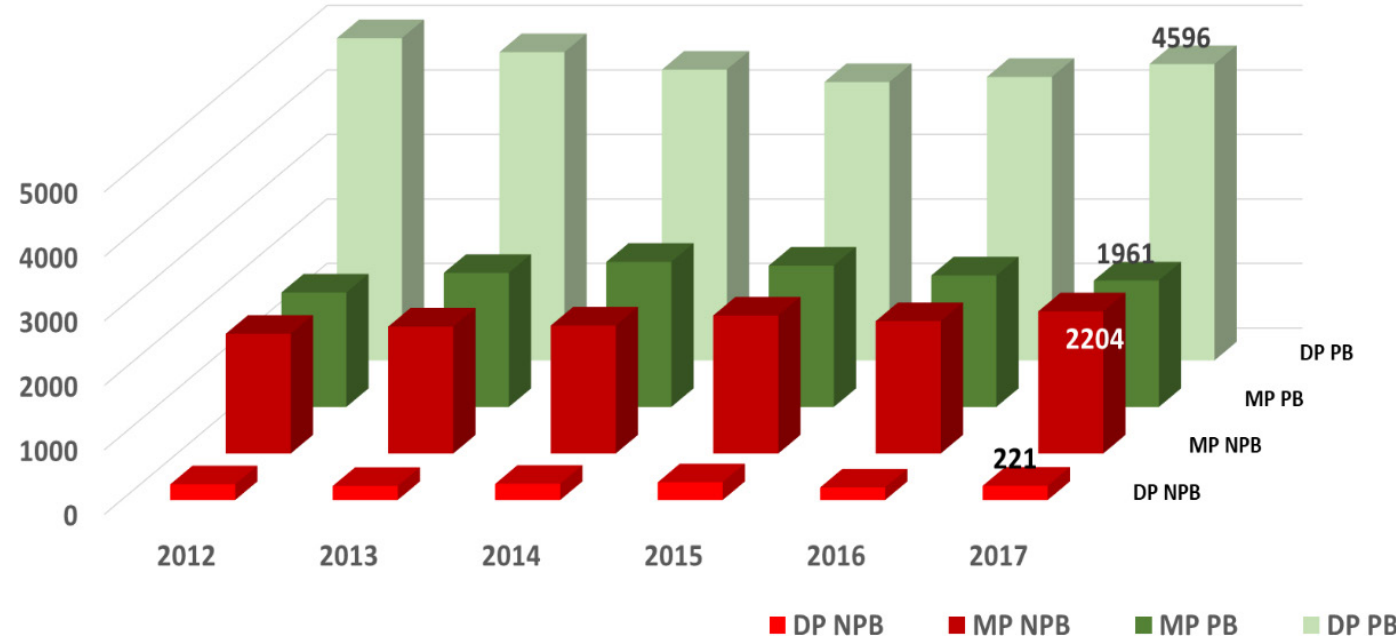

DP PB: Distinta patología. Procede baja. MP PB: Misma patología. Procede baja. MP NPB: Misma patología. No procede baja. DP NPB: Distinta patología. No procede baja.

En el año que hemos tomado de referencia (2017) se denegaron 2.204 bajas solicitadas por el trabajador en los Centros de Atención e Información de la Seguridad Social (CAISS) o bien emitidas de forma indebida por el médico de atención primaria. Lo que representa un $24,5 \%$ de bajas denegadas con respecto a las 8.982 instadas, porcentaje que se ha venido manteniendo a lo largo del tiempo.

Las bajas confirmadas que habían sido emitidas por el médico de atención primaria por distinta patología con anterioridad a 180 días han representado el $51 \%$ de los casos acontecidos.

De esta forma queda objetivado que el control de las recaídas de incapacidad temporal en los 6 meses siguientes a la emisión de altas por los inspectores médicos adscritos al INSS ha resultado una estrategia útil de gestión de la IT.

Es de interés señalar que generalmente se han citado a reconocimiento médico aquellos casos que o bien han sido por solicitud del interesado, o bien cuando en la valoración previa sobre historia clínica del trabajador que ha realizado el inspector médico, la información encontrada no ha sido suficiente para tomar una decisión. En todo caso cualquier denegación de baja se ha realizado tras reconocimiento médico.

En segundo lugar, se destacan determinados aspectos en cuanto a las bajas médicas por recaída contempladas en el artículo 170.2. En el año 2017 de los 142.060 procesos revisados por superar los 365 días en situación de incapacidad temporal en España, en un $17,6 \%$ (25.048 casos) se emitió el alta médica por el INSS $^{21}$.

Se han contabilizado 19.659 recaídas (con frecuencia varias recaídas corresponden a un único proceso inicial), de las cuales, se ha denegado la baja médica a 5.576 solicitudes de incapacidad temporal $(28,4 \%)$ bien sea a través del Equipo de Valoración de Incapacidades, o bien por denegación directa en consulta del inspector médico del INSS ${ }^{22}$.

En estos casos nuevamente no ha sido preciso molestar o llamar a reconocimiento médico a aquellos trabajadores en los que la situación de incapacidad temporal era adecuada conforme a los datos médicos disponibles.

En tercer lugar, se destaca que en el año 2017 se han revisado 6.549 casos en virtud de haber sido considerados como recaídas enmarcadas en el artículo 174 de la LGSS (proceso de baja médica posterior a la denegación de incapacidad permanente en los 6 meses previos). En 2.393 (36,5\%) de estos casos revisados, el Equipo de Valoración de Incapacidades no ha encontrado limitaciones para la actividad laboral habitual. 


\section{APLICACIÓN DE LA INTELIGENCIA ARTIFICIAL (IA) AL CONTROL DE LOS PROCESOS DE INCAPACIDAD TEMPORAL EN EL SIGLO XXI. LA VARIABLE "RECAÍDA"}

El control de las recaídas de incapacidad temporal en el siglo XXI no es ajeno a la aplicación de herramientas diversas como la IA. Se puede plantear la existencia de una variable "recaída" en función del modelo matemático empleado (regresión logística, random forest $\mathrm{u}$ otro sistema de análisis multivariante). En todo caso se hace imprescindible contar con sistemas inteligentes de gestión del control de bajas de incapacidad temporal, que contemplen la legislación de incapacidad temporal.

Se acepta habitualmente que la IA es la capacidad de un sistema para interpretar correctamente datos externos, para aprender de dichos datos y emplear esos conocimientos para lograr tareas y metas concretas a través de la adaptación flexible.

Los diversos documentos electrónicos que constituyen el expediente de cada proceso de incapacidad temporal aportan a la base de datos del INSS una gran multiplicidad de datos tales como el código diagnóstico, código nacional de ocupación, contingencia, situación de recaída y otras informaciones propias de seguridad social.

Destaca el conocimiento tanto del código de enfermedad que se recoge en función de lo establecido en la Clasificación Internacional de Enfermedades (CIE 9, $9^{a}$ edición o CIE 10 en su caso) como del código de ocupación en función de lo establecido en el Real Decreto 1591/2010, de 26 de noviembre, que incluye la Clasificación Nacional de Ocupaciones (CNO).

Así mismo al INSS llega información desde el propio sistema de seguridad social sobre el código de la clasificación nacional de actividades económicas (CNAE) de España, dispuesta en el Real Decreto 475/2007, de 13 de abril de 2007, que permite la clasificación y agrupación de las unidades productoras según las actividades que se ejercen de cara a la elaboración de estadísticas.

Disponer de todos estos datos agregados ha sido fundamental para elaborar, a través de herramientas estadísticas, instrumentos de ayuda en el control médico de la incapacidad temporal como es el Manual de Tiempos Óptimos de Incapacidad Temporal, formulándose un tiempo medio ideal requerido para la resolución de un proceso clínico que ha originado una incapacidad para el trabajo habitual en un trabajador de una edad y ocupación determinada, utilizando las técnicas de diagnóstico y tratamiento normalizadas y aceptadas por la comunidad médica y asumiendo el mínimo de demora en la asistencia sanitaria del trabajador ${ }^{23}$.

Otros datos epidemiológicos sobre pensiones de incapacidad han permitido aplicativos o programas que el Instituto Nacional de la Seguridad Social ha incorporado en los últimos años a la sede electrónica de la seguridad social, como ha sido el caso de la aplicación informática denominada ESTADISS, que permite obtener datos de las estadísticas de pensiones del Sistema de Seguridad Social.

Se han llegado a publicar reseñas provenientes de las bases de datos corporativas del INSS, como datos absolutos de frecuencia en la prestación de IT, tales como que el lumbago (724.2 CIE 9) es el diagnóstico específico inicial más frecuente en los procesos de incapacidad temporal. Así mismo, que el grupo diagnóstico más frecuente es el de enfermedades del sistema osteomioarticular y del tejido conectivo. El grupo de diagnósticos de mayor duración media son las neoplasias. Siguiendo la Clasificación Nacional de Ocupaciones y superados los 365 días de duración de incapacidad temporal es el grupo de trabajadores de servicios, restauración, personales, protección y vendedores de comercio, el que acumula mayor número de procesos de incapacidad temporal ${ }^{23}$.

En la actualidad el Big data, en relación a la enorme cantidad de datos que maneja la seguridad social, ha desafiado a sus organizaciones para llegar a un uso inteligente de 
los mismos, renovando los procedimientos clásicos utilizados hasta ahora para buscar patrones repetitivos dentro de esos datos.

En relación con el Big data, la estadística analítica pretende llegar a conclusiones sobre la distribución de una variable (por ejemplo "el parte de alta médica") y sus asociaciones en una población (por ejemplo, la población que se encuentra de baja médica) a partir de la distribución de esa misma variable y sus asociaciones en una muestra extraída de ella. De esta forma la estadística analítica permite realizar hipótesis sobre variables sociosanitarias y laborales de especial significación en las prestaciones de seguridad social.

Una de las primeras líneas impulsadas por el INSS ha consistido en diseñar y aplicar los primeros modelos que permiten optimizar la actividad de control médico del INSS evitando citar a reconocimiento médico a aquellos trabajadores en situación de incapacidad temporal con criterio médico justificado y que no han requerido por tanto ser convocados a control médico por el INSS con anterioridad a los plazos legalmente establecidos.

Estos modelos generados con la herramienta SAS en la Seguridad Social son ya esenciales para indicar aquellas situaciones de incapacidad temporal que requieren un control médico de la prestación por la entidad gestora a través de la inspección médica del INSS.

Estos algoritmos se han integrado en ATRIUM (aplicativo de trabajo informático de las unidades médicas del INSS) y se vienen aplicando desde entonces en la actividad diaria de todas las direcciones provinciales del INSS, evitando así citaciones innecesarias y aumentando la eficiencia de los reconocimientos médicos realizados.

Han posibilitado además diseñar y ejecutar dos planes trimestrales de intensificación en el control de la incapacidad temporal de octubre a diciembre de 2018 y de marzo a mayo de 2019 que han sido esenciales para lograr una línea ascendente tanto en la racionalización como en la efectividad de los controles que se realizan, llegándose a traducir en más de un $30 \%$ de trabajadores reincorporados nuevamente a la actividad laboral sobre el total de los reconocimientos realizados ${ }^{24}$.

La utilización de estos modelos de estadística analítica en la lucha contra la utilización indebida de las prestaciones ha merecido impulso y reconocimiento desde la Agencia Internacional de la Seguridad Social ${ }^{24}$.

El análisis de los primeros resultados ha supuesto nuevas evidencias para mejorar la eficacia del diseño de nuevos modelos que continúa desarrollando el INSS con las herramientas de IA que ha puesto a su disposición la Gerencia Informática de la Seguridad Social.

No resulta difícil aventurar que en estos primeros modelos la posible variable "recaída" y la variable "número de recaídas" tienen ya un inestimable valor entre las variables de definen un proceso de incapacidad temporal a la hora de detectar casos que no obedezcan a las frecuencias esperables.

\section{LARECAÍDAENINCAPACIDADTEMPORALFRENTEALAREINCORPORACIÓN LABORAL}

Por último, es relevante recordar que para disminuir la existencia de procesos de recaída y de alargamientos, muchas veces innecesarios, del periodo de incapacidad temporal, se ha señalado la importancia del desarrollo de políticas que faciliten la reincorporación laboral después de situaciones de baja, sobre todo tras procesos de baja de larga duración Estas políticas sin duda beneficiarían el anticipo temporal en la reincorporación laboral.

Algunos autores vienen propugnado la posible alta médica parcial tras una baja (reduciendo exigencias para facilitar incorporación). Hay que favorecer la adecuación de 
las situaciones de incapacidad médica larga y la adaptación en el retorno al trabajo, así como posibilitar la reintegración paulatina del trabajador con "Altas Parciales» que faciliten el reingreso al trabajo y prevengan recaídas. Más allá del alta médica laboral por curación o mejoría hay que facilitar la incorporación gradual al trabajo y el alta parcial lo permitiría en condiciones pactadas favoreciendo la reintegración laboral paulatina con un acuerdo entre las partes: el médico que controla o sigue al paciente, el trabajador y su empresa. Incluiría un documento con recomendaciones sobre qué tareas puede realizar si se incorpora al trabajo o si se puede beneficiar de prácticas como un cambio o reducción de horario o de tareas hasta que se consiga la recuperación total ${ }^{25}$.

Una apuesta legislativa interesante para estimular la reincorporación laboral la podemos encontrar en la Resolución de 28 de febrero de 2019, de la Secretaría de Estado de Función Pública, por la que se dictan instrucciones sobre jornada y horarios de trabajo del personal al servicio de la Administración General del Estado y sus organismos públicos $^{26}$.

En el punto 8 de esta Resolución se expone que los empleados o empleadas públicos que se reincorporen al servicio efectivo a la finalización de un tratamiento de radioterapia o quimioterapia podrán solicitar una adaptación progresiva de su jornada de trabajo ordinaria. La Administración podrá conceder esta adaptación cuando la misma coadyuve a la plena recuperación funcional de la persona o evite situaciones de especial dificultad o penosidad en el desempeño de su trabajo. Esta adaptación podrá extenderse hasta un mes desde el alta médica y podrá afectar hasta un $25 \%$ de la duración de la jornada diaria, preferentemente en la parte flexible de la misma, considerándose como tiempo de trabajo efectivo. La solicitud irá acompañada de la documentación que aporte la persona interesada para acreditar la existencia de esta situación, y la Administración deberá resolver sobre la misma en un plazo de tres días, sin perjuicio de que, para comprobar la procedencia de esta adaptación, la Administración podrá recabar los informes del Servicio de Prevención de Riesgos Laborales o de cualesquiera otros órganos que considere oportuno sobre el tratamiento recibido o las actividades de rehabilitación que le hayan sido prescritas.

El plazo al que se refiere el párrafo anterior podrá ampliarse en un mes más cuando el empleado o la empleada pública justifiquen la persistencia en su estado de salud de las circunstancias derivadas del tratamiento de radioterapia o quimioterapia.

Con carácter excepcional, y en los mismos términos indicados, esta adaptación de jornada podrá solicitarse en procesos de recuperación de otros tratamientos de especial gravedad, debiendo en este supuesto analizarse las circunstancias concurrentes en cada caso.

Aunque los plazos legales de un mes, anteriormente citados, para conseguir la reincorporación laboral completa y evitar recaídas innecesarias, pueden resultar demasiado cortos o rígidos, con la adecuada supervisión del servicio de prevención de la empresa, podrían alargarse en determinados casos y convertirse en una alternativa útil en la lucha contra el absentismo laboral derivado del uso excesivamente prolongado de las bajas laborales que padece hoy el sistema de seguridad social.

En materia de vigilancia de la salud, la actividad sanitaria de los servicios de prevención de las empresas deberá abarcar, en las condiciones fijadas por el artículo 22 de la Ley 31/1995, de Prevención de Riesgos Laborales ${ }^{27,28}$ :

1. ${ }^{\circ}$ Una evaluación de la salud de los trabajadores inicial después de la incorporación al trabajo o después de la asignación de tareas específicas con nuevos riesgos para la salud.

2. ${ }^{\circ}$ Una evaluación de la salud de los trabajadores que reanuden el trabajo tras una ausencia prolongada por motivos de salud, con la finalidad de descubrir sus eventuales orígenes profesionales y recomendar una acción apropiada para proteger a los trabajadores.

3. ' Una vigilancia de la salud a intervalos periódicos. 
En esta línea las personas que se reincorporan al trabajo después de una situación de incapacidad temporal deben ser sometidas a un especial control por el servicio de prevención y si fueran considerados trabajadores especialmente sensibles entrarían en el objetivo de actuaciones concretas.

El personal sanitario del servicio de prevención estudiará y valorará, especialmente, los riesgos que puedan afectar a las trabajadoras en situación de embarazo o parto reciente, a los menores y a los trabajadores especialmente sensibles a determinados riesgos, y propondrá las medidas preventivas adecuadas ${ }^{27.28}$.

\section{ALGUNAS CONCLUSIONES EN MATERIA DE INCAPACIDAD TEMPORAL Y SUS RECAÍDAS}

1. Las recaídas de incapacidad temporal después de las altas médicas emitidas por el INSS se encuentran reguladas en la legislación española de seguridad social y han sido motivo de diversos criterios jurisprudenciales.

2. Las recaídas de incapacidad temporal obedecen a periodos de incapacidad temporal que no se distancian más de 6 meses en el tiempo y son consecuencia de la misma enfermedad.

3. Las recaídas de procesos que inicialmente han sido cerrados por alta médica emitida por el Instituto Nacional de la Seguridad Social están sometidos a especiales requerimientos reglamentarios que consisten en un sistema de inspección directa llevada a cabo por los inspectores médicos adscritos al INSS, así como por los Equipos de Valoración de Incapacidades.

4. Las modificaciones legislativas en cuanto a recaídas de la situación de incapacidad temporal y la competencia para su determinación son motivo de continuas precisiones legislativas. La última modificación del artículo 170.1 de la Ley General de la Seguridad Social vuelve a incidir en esta materia en diciembre de 2018 aumentado las competencias del Instituto Nacional de la Seguridad: "el Instituto Nacional de la Seguridad Social ejercerá, a través de los inspectores médicos adscritos a dicha entidad, las mismas competencias que la Inspección de Servicios Sanitarios de la Seguridad Social u órgano equivalente del respectivo servicio público de salud, para emitir un alta médica a todos los efectos, así como para considerar que existe recaída en un mismo proceso cuando se produzca una nueva baja médica por la misma o similar patología dentro de los ciento ochenta días naturales siguientes a la fecha de efectos del alta médica anterior".

5. Desde el año 2013 hasta el año 2019 se viene produciendo una elevación importante de los índices que reflejan la situación de uso de la prestación de incapacidad temporal en nuestro país.

6. Las recaídas afectan directamente a la delimitación de la duración máxima del subsidio de incapacidad temporal en cada trabajador, pues facilitan alcanzar dicha duración máxima del subsidio cuando se cumplen las condiciones para que un proceso de incapacidad temporal sea recaída de otro previo.

7. El reconocimiento de la existencia de recaída determina la entidad pagadora del subsidio en dicha situación.

8. En el año 2017 el $24,5 \%$ del total de los procesos de recaída de trabajadores cuyas incapacidades temporales habían sido finalizados por alta por inspección médica del INSS, y que fueron tramitados por los propios inspectores médicos del INSS, resultaron en una situación de denegación de baja médica, al considerar que estaban aptos para su trabajo y que padecían igual o similar patología y por tanto similar situación funcional con la que se emitió el alta.

9. La inteligencia artificial y el big data son herramientas de análisis estadístico para la obtención de modelos capaces de detectar aquellos casos de incapacidad temporal que más difieren del curso biológico esperable de los mismos 
atendiendo a sus particulares características y que pudieran además ser tributarias de alta médica al ser sometidas a control médico por los inspectores médicos adscritos al INSS.

10. La variable "recaída" puede ser utilizada con distintos "pesos específicos" en los modelos de inteligencia artificial diseñados a partir de herramientas de estadística predictiva con la finalidad de la mejora del control de los procesos de incapacidad temporal.

11. La Asociación Internacional de Seguridad Social impulsa y reconoce el uso de estos modelos en la lucha contra el uso indebido de prestaciones de Seguridad Social.

12. La colaboración de los servicios médicos de las empresas en la reincorporación al trabajo de trabajadores tras una ausencia prolongada por baja médica, puede ser una opción para desarrollar, con los oportunos mecanismos de colaboración, actuaciones que eviten determinadas recaídas en la situación de IT.

\section{BIBLIOGRAFÍA}

1. Laín Entralgo, P. Historia de la Medicina. $1^{\text {a }}$ Ed, reimpresión 2001. Masson SA Barcelona (página 673).

2. Arancón Viguera, Ángel. Teoría y Práctica de la Medicina Evaluadora. 2004. Editorial Mapfre. Madrid (páginas 4-6).

3. Ramazzini, Bernardo. Tratado de las Enfermedades de los artesanos. NIPO: 354-03-004-3. Instituto de Salud Carlos III, 1999. Madrid (página 254)

4. Incapacidad Temporal. Manual para el manejo en atención primaria. Grupo Lex Artis. Sociedad Madrileña de Medicina Familiar y Comunitaria. ISBN 978-84-612-7648-6. Edita Sociedad Madrileña de Medicina Familiar y Comunitaria con la colaboración de AMAT. 2008 (página 17 y siguientes).

5. Presupuestos de la Seguridad Social ejercicio 2019. Consultado (página 616) el 27.04.2019 en: http:// www.seg-social.es/wps/wcm/connect/wss/3187c9a6-0e4d-419b-a9f7-ea99bf3ce4e8/SVT01PRO2018P. pdf?MOD=AJPERES\&CVID $=$

6. Contabilidad Nacional Trimestral de España: principales agregados. Cuarto trimestre de 2018. Documento consultado el 27.04.2019 en: http://www.ine.es/daco/daco42/daco4214/cntr0418.pdf

7. Incapacidad Temporal. Encrucijada ética, clínica y de gestión. Grupo de Trabajo de la Sociedad Española de Medicina Familiar y Comunitaria. 1999. SEMFYC. Barcelona. Páginas 4, 18, 20

8. Datos de incapacidad temporal 2018. Agregado del sistema de seguridad social. Incapacidad temporal. Contingencias Comunes, excepto autónomos. Datos acumulados a mes de diciembre de 2018. Duración media de los procesos, incidencia media mensual por cada mil trabajadores protegidos. Número medio mensual de procesos iniciados. En: http://www.seg-social.es/wps/wcm/connect/wss/7d70588d-b2ff-41538b4e-5f2dacb93f5a/2018_SIS_COMUNES_DUR_1.pdf?MOD=AJPERES\&CONVERT_TO=linktext\&ContentC ache $=$ NONE $\&$ CACHE $=$ NONE $\&$ CACHEID $=$ ROOTWORKSPACE.Z18_9H5AH880M8TN80QOV0H20V0000 7d70588d-b2ff-4153-8b4e-5f2dacb93f5a-mV-efPi

9. Datos de incapacidad temporal 2013. Agregado del sistema de seguridad social. Incapacidad temporal. Contingencias Comunes, excepto autónomos y hogar. Datos acumulados a mes de diciembre de 2013. Duración media de los procesos, incidencia media mensual por cada mil trabajadores protegidos. Número medio mensual de procesos iniciados. En: http://www.seg-social.es/wps/wcm/connect/wss/7437de89de23-4a03-a289-d841a020bca8/175478.pdf?MOD=AJPERES\&CONVERT_TO=linktext\&ContentCache $=\mathrm{N}$ ONE $\&$ CACHE $=$ NONE $\&$ CACHEID =ROOTWORKSPACE.Z18_9H5AH880M8TN80QOV0H20V00007437de89-de23-4a03-a289-d841a020bca8-mfRbK9x

10. VII informe ADECCO sobre absentismo laboral. Consultado el 16.06.2019 en: https://www.adeccogroup. es/wp-content/uploads/2018/06/NdP-La-tasa-de-absentismo-en-España-alcanza-su-máximohistórico-un-5.pdf

11. Real Decreto Legislativo $1 / 1995$, de 24 de marzo, por el que se aprueba el texto refundido de la Ley del Estatuto de los Trabajadores. «BOE» núm. 75, de 29/03/1995. Referencia: BOE-A-1995-7730. Consultado el 16.06.2019 en: https://www.boe.es/eli/es/rdlg/1995/03/24/1/con

12. Ley $35 / 2010$, de 17 de septiembre, de medidas urgentes para la reforma del mercado de trabajo. "BOE" núm. 227, de 18/09/2010. Referencia: BOE-A-2010-14301. Consultado en: https://www.boe.es/eli/ es/1/2010/09/17/35/con 
13. Real Decreto Legislativo $8 / 2015$, de 30 de octubre, por el que se aprueba el texto refundido de la Ley General de la Seguridad Social. Ministerio de Empleo y Seguridad Social. «BOE» núm. 261, de 31 de octubre de 2015. Referencia: BOE-A-2015-11724. Consultado en: https:/www.boe.es/eli/es/rdlg/2015/10/30/8/con

14. López Insúa, Belén del Mar. Tesis doctoral. La incapacidad temporal como contingencia protegida por la seguridad social. Dirección: Prof. Dr. José Luis Monereo Pérez. Granada noviembre 2012. ISBN: 978-849028-479-7. Página 319 y siguientes.

15. Antonio Vicente Sempere Navarro, José Luis Tortuero Plaza, Rodrigo Martín Jiménez, Jordi García Viña. 21.05.2007. Hacia un nuevo modelo de gestión de la incapacidad laboral. Investigación realizada por el Grupo de Investigación sobre El control de la incapacidad temporal. Subvención dirigida a actividades de estudio e investigación en el ámbito de la protección social (FIPROS 2005). Ministerio de Trabajo y Asuntos Sociales. Orden TAS/1051/2005, de 12 de abril.

16. Real Decreto-ley $28 / 2018$, de 28 de diciembre, para la revalorización de las pensiones públicas y otras medidas urgentes en materia social, laboral y de empleo. Disposición final segunda, punto cinco. BOE número 314 de 29 de diciembre de 2018. Referencia: BOE-A-2018-17992. Consultado el 24.05.2019 y el 30.05.2019 en: https://www.boe.es/eli/es/rdl/2018/12/28/28/con

17. Orden de 13 de octubre de 1967 por la que se establecen normas para la aplicación y desarrollo de la prestación por incapacidad laboral transitoria en el Régimen general de la Seguridad Social. (Texto consolidado con Última modificación: 20 de junio de 2015). «BOE» núm. 264, de 4 de noviembre de 1967, páginas 15166 a 15170 (5 págs.) Referencia: BOE-A-1967-19566. Consultado en: https://www.boe.es/eli/es/o/1967/10/13/(1)/con

18. Orden de 21 de marzo de 1974, por la que se regulan determinadas funciones de la Inspección de Servicios Sanitarios de la Seguridad Social en materia de altas médicas. "BOE» núm. 94, de 19 de abril de 1974, páginas 7993 a 7994 (2 págs.). Sección: I. Disposiciones generales. Departamento: Ministerio de Trabajo. Referencia: BOE-A-1974-646. Consultado en: https://www.boe.es/boe/dias/1974/04/19/pdfs/ A07993-07994.pdf

19. Real Decreto $625 / 2014$, de 18 de julio, por el que se regulan determinados aspectos de la gestión y control de los procesos por incapacidad temporal en los primeros trescientos sesenta y cinco días de su duración. «BOE» núm. 176, de 21 de julio de 2014. Referencia: BOE-A-2014-7684. Consultado en: https://www.boe. es/eli/es/rd/2014/07/18/625/con

20. Informe Estadístico 2011 consultado el 21.04.2019 en la dirección web: http://www.seg-social.es/wps/ wcm/connect/wss/435e4f12-1c9d-45c9-a7da-1f171edfb029/INFORME+ESTAD\%C3\%8DSTICO.2011on+1 ine.pdf?MOD=AJPERES $\&$ CVID $=$

21. Página 52 del Informe Estadístico del INSS 2017 consultado el 20.04.2019 y 10.06.2019 en la dirección web: http://www.seg-social.es/wps/wcm/connect/wss/0542ae8b-24fd-4387-bd5c-52c80e12770e/Informe + estad\%C3\%ADstico+2017_INSS.pdf?MOD=AJPERES\&CVID=

22. Ponencia: "Valoración Médica y control de la Incapacidad temporal" Dr. Luis Sánchez Galán. Subdirector General de Coordinación de Unidades Médicas del INSS. JORNADA DE INCAPACIDAD TEMPORAL: GESTIÓN DE LA INCAPACIDAD TEMPORAL EN LA ATENCIÓN PRIMARIA DE SALUD. Miércoles 30.05.2018. Hospital Son Llàtzer (Palma de Mallorca)

23. Datos epidemiológicos en incapacidad temporal en el Instituto Nacional de la Seguridad Social. Med Segur Trab (Internet) 2016; Suplemento extraordinario: 31-34. Luis Sánchez Galán. Última consulta realizada el día nueve de junio de 2019 en: http://gesdoc.isciii.es/gesdoccontroller?action=download \&id=13/09/2016-cdb46c85d0

24. El INSS obtiene un reconocimiento internacional por su plan para el refuerzo del control de la Incapacidad Temporal. Consultado el 15.06.2019 en: https://revista.seg-social.es/2019/06/10/el-inss-obtiene-unreconocimiento-internacional-por-su-plan-para-el-refuerzo-del-control-de-la-incapacidad-temporal/

25. Medicina y Seguridad del Trabajo versión On-line ISSN 1989-7790 versión impresa ISSN 0465-546X Med. Segur. Trab. vol.63 no.246 Madrid ene. /mar. 2017. Retorno al trabajo tras cáncer de mama. Return to Work after Breast Cancer. Araceli López-Guillén García1, José Manuel Vicente Pardo2. Consultado el 14.06.2019 en: http://scielo.isciii.es/scielo.php?script=sci_arttext\&pid=S0465-546X2017000100051.

26. Resolución de 28 de febrero de 2019, de la Secretaría de Estado de Función Pública, por la que se dictan instrucciones sobre jornada y horarios de trabajo del personal al servicio de la Administración General del Estado y sus organismos públicos. Publicado en: «BOE» núm. 52, de 01/03/2019. Referencia: BOE-A2019-2861. Consultado en: https://www.boe.es/eli/es/res/2019/02/28/(1)/con

27. Ley 31/1995, de 8 de noviembre, de prevención de Riesgos Laborales. "BOE» núm. 269, de 10 de noviembre de 1995. Referencia: BOE-A-1995-24292. Artículo 22. Consultado en: https://www.boe.es/buscar/ pdf/1995/BOE-A-1995-24292-consolidado.pdf

28. Real Decreto 39/1997, de 17 de enero, por el que se aprueba el Reglamento de los Servicios de Prevención. "BOE» núm. 27, de 31 de enero de 1997. Referencia: BOE-A-1997-1853. Artículo 37.3. Consultado en: https://www.boe.es/buscar/pdf/1997/BOE-A-1997-1853-consolidado.pdf 